\title{
Theory of Insulator Metal Transition and Colossal Magnetoresistance in Doped Manganites
}

\author{
T.V. Ramakrishnan * H. R. Krishnamurthy * \\ Centre for Condensed Matter Theory, Department of Physics, \\ Indian Institute of Science, Bangalore 560 012, India
}

(Dated: October 31, 2018)

\begin{abstract}
The persistent proximity of insulating and metallic phases, a puzzling characterestic of manganites, is argued to arise from the self organization of the twofold degenerate $e_{g}$ orbitals of $M n$ into localized Jahn-Teller(JT) polaronic levels and broad band states due to the large electron - JT phonon coupling present in them. We describe a new two band model with strong correlations and a dynamical mean-field theory calculation of equilibrium and transport properties. These explain the insulator metal transition and colossal magnetoresistance quantitatively, as well as other consequences of two state coexistence.
\end{abstract}

PACS numbers: 75.47.Lx, 75.47.Gk, 71.27.+a, 71.30.+h, 71.38.-k

Doped perovskite manganites $\operatorname{Re}_{1-x} A_{x} \mathrm{MnO}_{3}$, where $R e$ and $A$ are rare earth and alkaline earth ions, show a rich variety of electronic, magnetic and structural phenomena and phases 1, 2]. Unusual effects, such as insulator-metal(IM) transitions both as a function of $x$ and of temperature $T$ over a wide region $x \lesssim 0.5$, or even as a consequence of isotope substitution $\left(O^{18} \rightarrow O^{16}\right)[3]$, colossal magneto-resistance(CMR) near $T_{I M}$ and 'melting' of the charge/orbitally ordered insulator $(x \gtrsim 0.5)$ into a metal in a relatively small(5-7 Tesla) magnetic field all suggest that metallic and insulating phases are always very close in free energy. This is also reflected in the ubiquitous coexistence (static or dynamic) of two 'phases', one insulating with local lattice distortion and the other metallic without lattice distortion, with length scales varying from $10 A^{\circ}$ to $10^{3} A^{\circ}$ [2]

These phenomena are due to the dynamics of the $e_{g}$ electrons of $M n$ constrained by three strong on-site interactions, namely electron lattice or Jahn-Teller (JT) coupling which splits the twofold $e_{g}$ orbital degeneracy, ferromagnetic $e_{g}$ spin- $t_{2 g}$ spin exchange or Hund's coupling $J_{H}$ and $e_{g}$ electron repulsion $U$. The respective energies are $E_{J T} \simeq 0.5 \mathrm{eV}, J_{H} \simeq 2 \mathrm{eV}$ and $U \simeq 5 \mathrm{eV}$, compared to the $e_{g}$ electron intersite hopping $t \simeq 0.2 \mathrm{eV}$ which sets the kinetic energy scale [4]. Understanding their observed consequences is one of the major challenges in the physics of strongly interacting electrons. Earlier theoretical attempts neglect one or more of these strong interactions and make further approximations; the predictions do not agree with many characteristics of manganites. For example the ferromagnetic Curie transition in a number of manganites is from an insulator to a metal, for $0.2 \lesssim x \lesssim 0.5$. However, a theory with just Hund's coupling, due to Furukawa [5], finds only a metallic phase, while Millis, Muller and Shraiman [6], who additionally include the electron-JT phonon coupling $g$ but treat the JT distortions as static displacements, obtain a metalmetal transition crossing over to an insulator-insulator transition as $g$ increases.

We propose and implement here a new approach which incorporates the crucial effects of all the three interactions and is based on a new idea, namely that of coexisting JT polaronic and broad band $e_{g}$ states, which we believe is the key to manganite physics. The idea and some of its consequences are described and calculations based on a new two band model are then outlined.

We first discuss the effect of large JT coupling $g$ on the initially twofold degenerate $e_{g}$ orbitals at each lattice site. There is one superposition (labelled $\ell$ ) which, when singly occupied,leads to a polaronic state with local octahedral symmetry breaking $M n-O$ bond distortion and energy $-E_{J T}$. Its intersite hopping is reduced (for $\left(E_{J T} / \hbar \omega_{0}\right) \gg 1$ where $\omega_{0}$ is the JT phonon mode frequency) by the exponential Huang-Rhys [7] or phonon overlap factor $\eta \simeq \exp \left(-E_{J T} / 2 \hbar \omega_{0}\right)$. Since $\eta \simeq \exp (-5)$ for $\mathrm{LaMnO}_{3}$, and being a local quantity is not likely to change much on doping, the $\ell$ polaron bandwidth $2 D^{*} \sim 2 z t \eta \simeq k_{B}(125 K)$ is small, and is neglected in much of this paper. At each site, there is necessarily another, orthogonal state (labelled $b$ ). Its energy is zero on the fraction $x$ of sites where the $\ell$ electrons are not present(hole-sites), and is $\bar{U}=\left(U+2 E_{J T}\right)$ on the $\ell$ electron sites. The $b$ electron hops adiabatically $\left(\hbar t^{-1} \ll \omega_{0}^{-1}\right)$, in an annealed random medium of repulsive $\ell$ sites and (for a homogeneous orbital liquid) forms a band whose re-normalized bandwidth $2 D$ increases with $x$ as well as with $T^{-1}$ and $H$. For, the inhibition of $b$ hopping due to large $\bar{U}$ is reduced when there are more hole-sites, and that due to large $J_{H}$ is reduced when the $t_{2 g}$ spin order is enhanced. We show in this paper that the simultaneous presence of $\ell$ polaron states and the $b$ band, the change in the effective band width of the latter with $x, T, H$ etc. and the consequent change in their relative occupation (leading to an insulator eg for $E_{J T}>D$ and metal for $E_{J T}<D$ ) are the basis of many phenomena seen in manganites. Our picture requires the persistence of local $J T$ distortions well into the metallic regime, as 
indeed seen by direct probes of instantaneous $M n-O$ bond length [8]. The $\ell$ polaron motion is anti-adiabatic $\left(2 D^{*} \ll \hbar \omega_{0}\right)$ while the $b$ electron motion is adiabatic $\left(2 D \gg \hbar \omega_{0}\right)$; the respective bandwidths are exponentially small and of order the bare value. By contrast, in earlier theoretical work [6, 9] where the $J T$ distortion is treated as static, both $\ell$ and $b$ electron dynamics become adiabatic so that the bands are of comparable width, leading to consequences which disagree with experiment.

The system of correlated $\ell$ and $b$ states described above can be modelled by the Hamiltonian

$$
\begin{gathered}
H_{\ell b}=\left(-E_{J T}-\mu\right) \sum_{i, \sigma} \ell_{i \sigma}^{\dagger} \ell_{i \sigma}-\mu \sum_{i \sigma} b_{i \sigma}^{\dagger} b_{i \sigma} \\
-\bar{t} \sum_{\langle i j\rangle, \sigma}\left(b_{i \sigma}^{\dagger} b_{j \sigma}+h c\right)+\bar{U} \sum_{i \sigma} n_{\ell i \sigma} n_{b i \sigma}+H_{m}
\end{gathered}
$$

In Eq.(1), the $\ell$ polaron has energy $\left(-E_{J T}\right)$, the $b$ electrons hop between nearest neighbour sites with an effective amplitude $\bar{t}$, and $\bar{U}$ is the effective repulsion between $\ell$ polarons and $b$ electrons of the same spin at a particular site [10]. The common chemical potential is $\mu . H_{m}$ is the magnetic part involving the $e_{g}$ spins $\overrightarrow{s_{i}}$ and $t_{2 g}$ core spins $\vec{S}_{i}$, namely

$$
H_{m}=-J_{H} \sum_{i} \overrightarrow{s_{i}} \cdot \overrightarrow{S_{i}}-J_{F} \sum_{\langle i j\rangle} \overrightarrow{S_{i}} \cdot \overrightarrow{S_{j}}-\mu_{B} \sum_{i} \overrightarrow{S_{i}} \cdot \vec{H}(2)
$$

In addition to the familiar Hund's coupling $J_{H}$ and Zeeman coupling to an external field $\vec{H}$ (neglecting the relatively smaller term $\mu_{B} \overrightarrow{s_{i}} \cdot \vec{H}$ ) we have included in $H_{m}$ a new ferromagnetic nearest neighbour exchange $J_{F}$ between the $t_{2 g}$ core spins.

The new term arises from a virtual double-exchange process in which an $\ell$ electron at a site $i$ hops quickly(adiabatically) to a nearest neighbour site $j$ and back. In the limit of large $\bar{U}$ and $J_{H}$, and approximating the $t_{2 g}$ spins as classical, i.e., $\vec{S}_{i}=S \hat{\Omega}_{i}$ where $\hat{\Omega}_{i}$ are unit vectors, the energy shift to second order in $\bar{t}$ is $\left(\bar{t}^{2} / 2 E_{J T}\right) \frac{1}{2}\left(\hat{\Omega}_{i} \cdot \hat{\Omega}_{j}+1\right)\left(n_{\ell i}\left(1-n_{j}\right)+n_{\ell j}\left(1-n_{i}\right)\right)$. The energy denominator $2 E_{J T}$ arises from the unrelaxed JT distorted intermediate state, the $\frac{1}{2}\left(\hat{\Omega}_{i} \cdot \hat{\Omega}_{j}+1\right)$ factor from large $J_{H}$, and the occupancy dependent terms from large $\bar{U}$. We have further approximated this by the simple exchange term in Eq.(2), whence $J_{F} \simeq\left(\bar{t}^{2} /\left(2 E_{J T} S^{2}\right)\right) x(1-x)$.

The model Hamiltonian $H_{\ell b}$ (Eq.(1)) can be motivated starting from a lattice model with two $e_{g}$ orbitals per site 1, 2]. Let $a_{i \alpha}^{\dagger}$ create an electron in orbital $\alpha$ at site $i,\left(\right.$ with $|\alpha=1>\equiv| x^{2}-y^{2}>$ and $\mid \alpha=2>\equiv$ $\mid 3 z^{2}-r^{2}>$ say), locally coupled to JT lattice modes $\left(Q_{x i}, Q_{z i}\right) \equiv\left(Q_{i}, \theta_{i}\right)$ by the term $H_{J T}^{i}=g a_{i \alpha}^{\dagger} \vec{\tau}_{\alpha \beta} a_{i \beta} \cdot \vec{Q}_{i}$ where $\vec{\tau}$ are the Pauli matrices. The eigenvalues of $H_{J T}^{i}$ are $\pm g Q_{i}$, the corresponding electron creation operators being labelled $b^{\dagger}$ and $\tilde{\ell}^{\dagger}$. The effective lattice potential energies for single electron occupation of these states are
$(K / 2) Q_{i}^{2} \pm g Q_{i}$ where $K$ is the phonon force constant. The lower $(\ell)$ branch has a minimum at a static lattice distortion $Q_{0}=g / K$ and energy $-E_{J T}=-\left(g^{2} / 2 K\right)$. $\ell_{i}^{\dagger}$ in Eq.(1) are polaron creation operators given by $\hat{\eta}_{i} \tilde{\ell}_{i}^{\dagger}$ where $\hat{\eta}_{i}\left(\sim \exp \left(i Q_{0} P_{i} / \hbar\right)\right.$ where $P_{i}$ is the radial momentum conjugate to $Q_{i}$ ) are unitary Lang-Firsov 11] like displacement transformations. The exponential reduction $\eta$ in the hopping amplitude of $\ell$ arises essentially from their ground state average. (We neglect the small fluctuations with respect to this average). No lattice distortion or reduction in hopping is associated with the higher $(b)$ branch. The small leading intersite term $H_{\ell b}^{\prime} \sim \bar{t} \eta \sum_{\langle i j\rangle}\left(\ell_{i}^{\dagger} b_{j}+b_{i}^{\dagger} \ell_{j}+h c\right)$ describing $\ell-b$ hybridization is not included in Eq.(1); some consequences are discussed later. Thus for $\left(E_{J T} / \hbar \omega_{0}\right) \gg 1$, and neglecting $H_{l b}^{\prime}$, we are led to $\mathrm{Eq}(1)$. We have made further approximations which are realistic for a homogenous orbital liquid, by statistically averaging quantities which depend on the orbital admixture angle $\left\{\theta_{i}\right\}$, eg the intersite hopping and the nearest neighbour $M n-O$ bond correlations (short-long), so that $\bar{t}$ and $b_{i}^{\dagger}$ are to be regarded as averaged over $\left\{\theta_{i}\right\}$. This is reasonable for $0.2 \lesssim x \lesssim 0.5$ in most manganites, but poor for those other values of $x$ for which one has strong orbital correlations or long range orbital order. Finally, in this regime, the chemical potential $\mu$ is chosen such that

$$
\left\langle n_{\ell i}\right\rangle+\left\langle n_{b i}\right\rangle=\bar{n}_{\ell}+\bar{n}_{b}=(1-x)
$$

We have calculated the equilibrium and transport properties of $H_{\ell b}$ (Eq.(1)) using the dynamical mean field theory (DMFT) [12] which is exact in $d=\infty$, and is accurate in three dimensions if spatial correlations are not crucial. $H_{\ell b}$, being a generalized form of the FalicovKimball model [13] with additional spin interactions $H_{m}$ (Eq.(2)) and constraint Eq.(3), is exactly soluble in this limit 12]. We treat the $t_{2 g}$ core spins as classical as stated earlier, and their interaction in the Curie-Weiss mean field approximation, and work in the large $J_{H}$ limit (i.e. $\left.S J_{H} / \bar{t} \gg 1\right)$. The local self energy $\Sigma_{i i}\left(\omega^{+}\right)$of the $b$ electrons and the mean magnetization $m=\left\langle\hat{\Omega}_{i}\right\rangle$ are determined self-consistently. From these we calculate the spectral density or density of states (DOS) $\rho_{b}(\omega)$ of the $b$ electrons which determines their occupation relative to that of the $\ell$ state, the current-current correlation function relevant for the Kubo formula for the electrical conductivity, etc 14]. Most of our calculations use a semicircular bare DOS with a bare bandwidth $2 D_{o}[15$.

Our results for $\rho_{b}(\omega)$ for different values of $x$ and $T$ are shown in Fig.1. Since the effective bandwidth $2 D$ of the $b$ electrons decreases significantly as $x$ decreases (for any sizeable $\bar{U}$ ), the $b$ band bottom is above the $\ell$ level for small $x$ e.g $x=0.1$ (Fig.1a), and the low temperature state is an insulator, ferromagnetic because of $J_{F}$. (We ignore the anti-ferromagnetic super-exchange as it is dominated by the much larger $J_{F}$ for $x \gtrsim .1$ ). On 


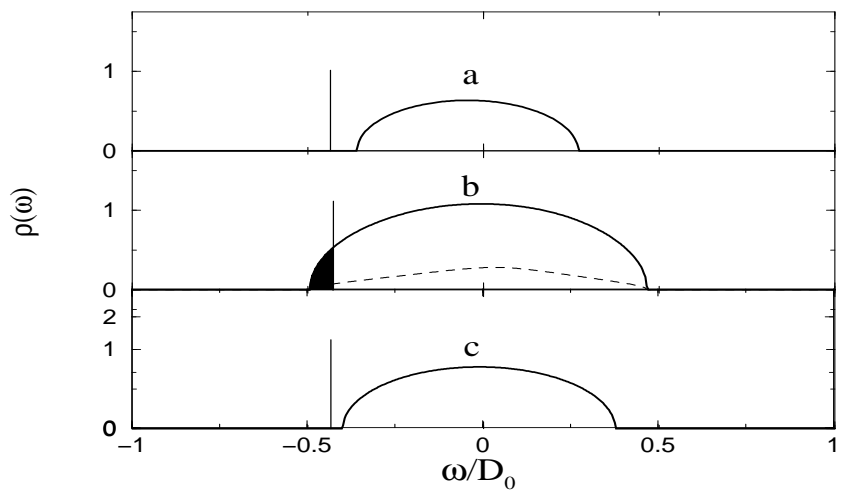

FIG. 1: Spectral density or $b$-DOS $\rho_{b}(\omega)$ for various values of doping $x$ and temperature $T$. The effective $\ell$ polaron level is marked as a vertical line. Parameters chosen are $E_{J T}=$ $-0.5 \mathrm{eV}, D_{o}=1.2 \mathrm{eV}, \bar{U}=5.0 \mathrm{eV} J_{F}=2.23 \mathrm{meV}$. (a) $x=0.1, T=0, \mu=-E_{J T}$; ferromagnetic insulator. (b) $x=0.3, T=180 K\left(<T_{c}=240 K\right)$; ferromagnetic metal. (c) $x=0.3, T=350 K$; paramagnetic insulator. Full(dotted) lines correspond to up(down) spin DOS. Occupied band states are shown shaded.

increasing $x, D$ increases as well, and beyond a critical $x_{c}$ for which $D=E_{J T}$, the low temperature state is a ferromagnetic metal, as in Fig.1b. We note that while both $b$ and $\ell$ states are occupied in the metal, most electrons are in the latter, polaronic state since $\bar{n}_{\ell}=0.66$ and $\bar{n}_{b}=0.04$. $T_{c}$ is largely due to $J_{F}$; for, $\bar{n}_{b}$ being very small, so is the $b$ electron contribution to $T_{c}$ via conventional double exchange. On increasing $T$ at this $x$, the $t_{2 g}$ spins disorder, reducing the effective $b$ electron hopping or $D$. Fig.1c shows the DOS at $T=350 K\left(>T_{c}\right)$. It is that of a small gap semiconductor. The metal insulator transition occurs very near $T_{c}$ because of the strong feedback between $D$ and the magnetization $m$. The carriers in the paramagnetic state are $b$ electrons excited across the relatively small effective band gap, not thermally unbound small polarons. The mobile carrier fraction $\bar{n}_{b}$ is very small in the metallic phase $\left(\bar{n}_{b} \simeq 0.06\right.$ for parameters corresponding to $\mathrm{La}_{0.7} \mathrm{Sr}_{0.3} \mathrm{MnO}_{3}$ ) and decreases rapidly [14] to a minimum at $T_{c}$, whereas the electron density is 0.7 per site. This is exactly the hitherto unexplained inference for $n_{e f f}$ from the observed Drude weight in this compound [16].

Our theory also describes transport properties fairly well. Fig.2 shows the electrical resistivity $\rho(T)$ versus $T$ for model parameters chosen to fit $T_{c}$ and $\rho\left(T_{c}\right)$ for $\mathrm{La}_{0.67} \mathrm{Ca}_{0.33} \mathrm{MnO}_{3}$ [17]. The results for semicircular DOS and tight binding DOS are nearly the same. We see clearly the sharp paramagnetic insulator to ferromagnetic metal transition, the former having a calculated effective electrical gap $\Delta_{\text {eff }}$ of $34 \mathrm{meV}$ while the experimental value is $48 \mathrm{meV}$ 18. The resistivity falls dramatically to about $2 \mathrm{~m} \Omega \mathrm{cm}$ just below $T_{c}$ and does not decrease much further thereafter, in contrast to the observed residual re-

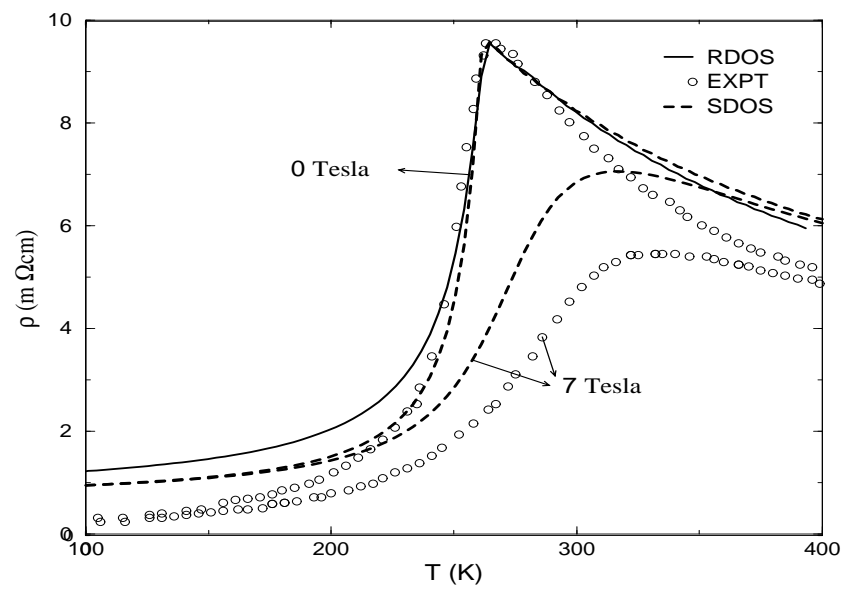

FIG. 2: The resistivity $\rho(T)$ of $L a_{1-x} C a_{x} M n O_{3}(x=0.33)$ as a function of temperature $T$. Calculated (full lines for realistic anisotropic hopping, dotted lines for a semicircular DOS) and experimental (circles, from ref. 17]) results for $H=0$ and 7 Tesla are shown. Parameters, chosen to fit $T_{c}$ and $\rho\left(T_{c}\right)$, are $E_{J T}=-0.5 \mathrm{eV}(\mathrm{SDOS}),-0.6 \mathrm{eV}(\mathrm{RDOS}), 2 D_{o}=2.4 \mathrm{eV}$ (SDOS), $2.44 \mathrm{eV}$ (RDOS), $\bar{U}=5 \mathrm{eV}$, and $J_{F}=2.37 \mathrm{meV}$.

sistivity values which can be as small as $50 \mu \Omega \mathrm{cm}$. This is a consequence of our neglect of inter-site $\ell$ coherence and is discussed later. The resistivity in a field of 7 Tesla is also shown in Fig.2. The colossal decrease(CMR) is apparent [18].

The properties of manganites vary strongly and characteristically with the ionic species $R e$ and $A$. This is natural in our model, since the relative balance between metal and insulator is strongly affected by small changes in $E_{J T}$ and $D_{o}$ and the carrier density depends exponentially on $\left(D-E_{J T}\right)$. For example, keeping $E_{J T}$ fixed (for a given $x=0.3$ say), and varying $2 D_{o}$, we find the variations in physical properties shown in Fig.3. $T_{c}$, being proportional to $J_{F}$, increases roughly as $D_{o}^{2}$. The Curie transition changes from insulator-insulator to insulator-metal to metal-metal, as is seen experimentally in the sequence $\mathrm{Pr}_{0.7} \mathrm{Ca}_{0.3} \mathrm{MnO}_{3}, \mathrm{La}_{0.7} \mathrm{Ca}_{0.3} \mathrm{MnO}_{3}$ and $\mathrm{La}_{0.7} \mathrm{Sr}_{0.3} \mathrm{MnO}_{3} . \mathrm{D}_{o}$ is believed [19] to increase in this order. We also exhibit the enormous variation in the calculated fractional magneto-resistance $\{\Delta R(H) / R(H)\}_{T=T_{c}}$ for $H=7$ Tesla as a function of $T_{c}$; the agreement with experiment [20] is very good.

Thus, in the regime $0.2<x<0.4$, a wide range of physical properties for a number of doped manganites can be understood physically and described quantitatively by our theory 14]. The ratio $\left(E_{J T} / D_{o}\right)$ broadly determines the systematics and $D_{o}$ the basic energy scale $\left(J_{H}\right.$ and $\bar{U}$ being close to $\infty$ for our purposes).

The intersite hopping of the $\ell$ polarons arising from term $H_{\ell b}^{\prime}$, with a characteristic temperature scale of $T^{*} \sim\left(z \eta \bar{t} / k_{B}\right) \simeq 125 \mathrm{~K}$, has many important consequences. Below $T^{*}$ the $\ell$ states can form a coherent band 


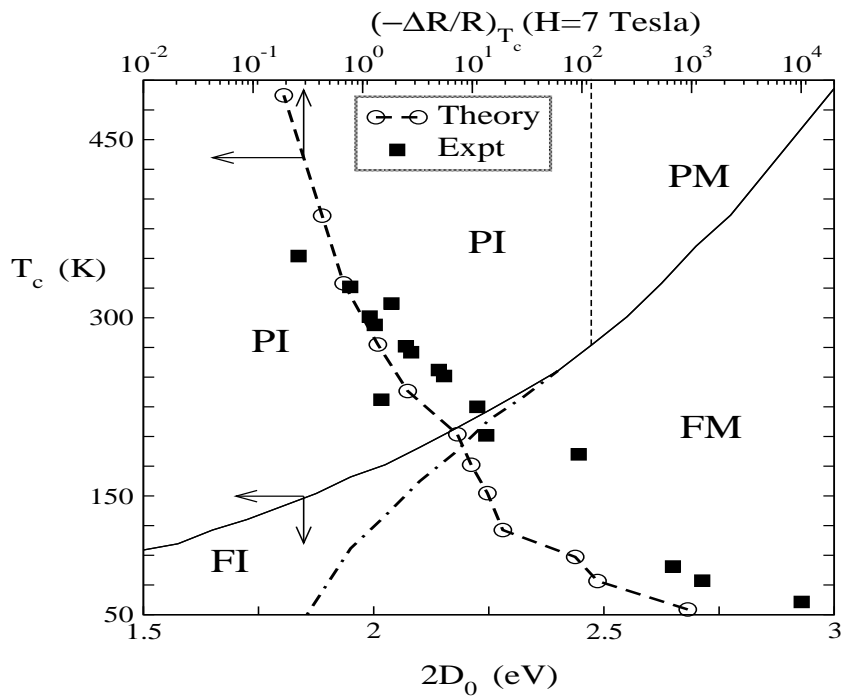

FIG. 3: Physical properties as a function of the $d$ electron bandwidth $2 D_{o}$, for fixed $E_{J T}=0.5 \mathrm{eV}$. Full line: ferromagnetic $T_{c}$ vs $D_{o}$. The resistive transition is from an insulator to an insulator(PI-FI) for small $D_{o}$, from an insulator to a metal(PI-FM) for intermediate $D_{o}$, and from a metal to a metal(PM-FM) for large $D_{o}$, as indicated . Broken line: calculated fractional magnetoresistance $(\Delta R(H) / R(H))_{T_{c}}$ vs $T_{c}$ for $H=7$ Tesla . Experimental points are from [20].

due to hybridization with $b$ states, whence the JT distortion can become dynamic (time scale $\sim \hbar / k_{B} T^{*}$ ), and also smaller self-consistently. The $b$ electron scattering and the consequent resistivity then vanish at $T=0$, and are nonzero only if static disorder is present. This can lead to a metallic state with a small residual resistivity or to an Anderson localized insulating state depending on the amount of disorder. The strong dependence of $T_{c}$ on isotope mass $M_{0}$ is also a consequence of $\ell$ polaron hopping, since its direct double exchange contribution to $T_{c}$ will be proportional to $\bar{t}(1-x) \exp \left(-E_{J T} / 2 \hbar \omega_{0}\right)$ and $\omega_{0}=\sqrt{K / M_{0}}$. The isotope effect thus estimated is of the right size 21].

Spatial correlations and inhomogeneities can be investigated in an extended version of the model in Eq.(1) by including the orbital angles $\theta_{i}$ as additional degrees of freedom. The intersite hopping $t_{i j}$ now depends on $\theta_{i}$ and $\theta_{j}$. There are a number of anharmonic, steric, elastic terms, eg., nearest neighbour long-short $M n-O$ bond correlations, coupling between JT modes and strain,etc., which depend on these angles as well. These, and other factors such as the variation of $E_{J T}$ with local ion size, can be included in Eq.(1), and questions such as short range correlation, orbital order, long range order in $\left(\bar{n}_{\ell i} \pm \bar{n}_{b i}\right)$ (total charge/relative charge order), disorder induced 'phase' separation, explored by going beyond the self-consistent single site DMFT used here. It would also be interesting to explore the idea of exponentially separated time scales and adiabatic - nonadiabatic crossover in other oxides and organic molecules with orbital degeneracy and strong local symmetry breaking Jahn Teller coupling.

We would like to acknowledge support from the Indo-French Centre for Promoting Advanced Research grant 2404-1 (HRK),US-India project ONR N 00014-970988(TVR) and the Council for Scientific and Industrial Research, India (SRH,GVP).

[*] Also Condensed Matter Theory Unit, JNCASR, Jakkur, Bangalore 560 064, India.

[†] Also Abdus Salam International Centre for Theoretical Physics, 11 Strada Costiera, Trieste 34014, Italy

[1] For reviews, see Colossal Magnetoresistance Oxides, ed Y Tokura (Gordon and Breach, New York, 2000); M B Salamon and M Jaime, Rev. Mod. Phys. 73, 583 (2001).

[2] eg. Nanoscale Phase Separation in Manganites by E Dagotto (Springer Verlag, New York and Heidelberg, 2002).

[3] N A Babushkina, et al., Nature 391, 159 (1998).

[4] see eg. D D Sarma et al., Phys. Rev. Lett. 75, 1126 (1995), S Satpathy, Z S Popovic, and F R Vukajlovic, Phys. Rev. Lett. 76, 960 (1996).

[5] N Furukawa, Journal of the Physical Society of Japan, 642734 (1995).

[6] A J Millis, R Mueller and B I Shraiman, Phys. Rev. B54, 5389, 5405 (1995).

[7] K Huang and F Rhys, Proc. Roy. Soc. London Ser. A 204, 406 (1950).

[8] see for example the pulsed neutron measurements of D. Louca et al., Phys. Rev. B. 56, R8475 (1997). and the EXAFS results of $\mathrm{C}$ Meneghini et al., J Phys. Cond. Matt. 14, 1967 (2002).

[9] M S Laad, L Craco and E Muller-Hartmann Phys. Rev. B 63, 214419 (2001).

[10] We neglect same site opposite spin interactions since such configurations are strongly disfavoured in the large $J_{H}$ limit we treat in this paper.

[11] G I Lang and A Yu Firsov, Soviet Physics JETP 43, 1843 (1962).

[12] A Georges, G Kotliar, W Krauth and M J Rozenberg, Rev. Mod. Phys. 68, 13 (1996).

[13] L. M. Falicov and J C Kimball, Phys. Rev. Lett. 22, 997 (1969).

[14] Details as well as additional new results will be published elsewhere.

[15] We find that the calculated results are nearly the same for semicircular DOS and realistic tight binding DOS (eg. see Fig.2 for resistivity).

[16] Y. Okimoto, et.al., Phys. Rev. B 55, 4206 (1997).

[17] G. J. Snyder et al., Phys. Rev. B 53, 14434(1996).

[18] The discrepancies can be reduced within our model by including short-range correlations which are neglected in the DMFT.

[19] H. Y. Hwang et. al., Phys. Rev.Lett. 75, 914 (1995).

[20] K Khazeni et al., Phys. Rev. Lett. 76, 295 (1996).

[21] G. M. Zhao, et al., Phys. Rev. B 63, 060402 (2001). 